\title{
Prognosis
}

\section{Personality disorders have a differential effect on the outcome of anxiety disorders}

\author{
Massion AO, Dyck IR, Shea T et al. Personality disorders and time to remission in generalized anxiety disorder, social \\ phobia and panic disorder. Archives of General Psychiatry 2002 May; 59: 434-40.

\section{QUESTION: What effect do personality disorders have on time to remission in people with generalised anxiety disorder, panic disorder or social phobia?}

Design

Multisite cohort study.

\section{Setting}

USA. Enrolment January 1989-November 1991 with 5 years of follow up.

\section{Participants}

514 participants in the Harvard/Brown Anxiety Research Program with at least one past or current episode of panic disorder with or without agoraphobia, generalised anxiety disorder or social phobia; a personality disorder assessment, and course data available for a least 6 months. Mean age 40 years; $66 \%$ women.

Sources of funding:

Not specified.

For correspondence:

A Massion, Department

of Psychiatry,

University of

Massachusetts Medical

School.annmassion@

umassmed.edu

\section{Assessment of risk factors}

Axis I disorders were diagnosed at study enrolment using a structured diagnostic instrument (SCALUP). Axis II disorders were assessed at 1 year follow up using the International Personality Disorder Examination. Major depression was controlled for.

\section{Main outcome measures}

Time to remission (defined as occasional mild symptoms or no symptoms for 8 consecutive weeks using Psychiatric Status Rating Scales).

\section{Main results}

People with avoidant personality disorder and dependent personality disorder had a lower likelihood of remission from generalised anxiety disorder (34\% and 14\% lower respectively). People with avoidant personality disorder had a $41 \%$ lower likelihood of remission from social phobia, but personality disorder did not predict panic disorder remission.

\section{Conclusions}

Personality disorders have a differential effect on the outcome of anxiety disorders. Personality disorders reduce the likelihood of remission in generalised anxiety disorder and social phobia, but not panic disorder.

\section{COMMENTARY}

There are few longitudinal studies of anxiety disorders. Previous work has been limited by small samples, short follow-up and retrospective or selfratings. The strength of this study lies in its prospective design, naturalistic setting, structured ratings and multiple assessments over a lengthy period. Nevertheless, participants often had comorbid anxiety disorders, the number with any single anxiety disorder or personality disorder was relatively low and personality disorder was assessed one year after enrolment so there could have been a state effect.

Studies such as this raise questions about the relationship between personality disorders and Axis I disorders. Does increased understanding of the biological underpinnings of personality, and the responsiveness of Axis I disorder to psychotherapy, mean that distinctions between Axis I and II conditions are based primarily on convenience rather than aetiological or therapeutic grounds? Given the chronicity of generalised anxiety disorder (which precedes a range of other disorders), some might argue that this condition ought to fall on Axis II. The presence of comorbid Cluster $\mathrm{C}$ personality disorders such as avoidant or dependent personality disorder would then conceivably point to a broader and apparently more refractory syndrome. Chronic anxiety symptoms and avoidant and dependent personality traits might share mediating psychobiological features.

The validity of distinctions between social phobia, particularly generalised social phobia, and avoidant personality disorder has similarly been questioned. People with avoidant personality disorder, for example, may respond to pharmacotherapy for social phobia. If avoidant personality traits in social phobia are a marker of a broader and more refractory disorder, it would be unsurprising that comorbid avoidant personality disorder predicted reduced remission in social phobia.

The finding that panic disorder is not influenced by personality disorder would strike some as at odds with clinical experience. Anecdotally, many clinicians have patients with borderline personality disorder where comorbid panic disorder had an apparently refractory course. The authors note that few participants had certain individual personality disorders and concede that this finding may reflect a type II error.

There is increasing focus on remission as a treatment goal in mood and anxiety disorders. This study raises the issue of whether people with anxiety disorder and comorbid personality disorders respond to earlier, more rigorous intervention. Further work is needed to answer this question, but the effect of personality disorders on anxiety disorder outcomes suggests that it may be useful to evaluate Axis II disorders in people with anxiety disorder and to address these during management. 